\title{
Is global hypomethylation a nidus for molecular pathogenesis of age-related noncommunicable diseases?
}

\author{
Apiwat Mutirangura*,1 \\ ${ }^{1}$ Center of Excellence in Molecular Genetics of Cancer \& Human Diseases, Department of Anatomy, Faculty of Medicine, \\ Chulalongkorn University, Bangkok, Thailand \\ *Author for correspondence: mapiwat@chula.ac.th
" a technique adding IRS methylation, such as Alu siRNA transfection, may be useful for treating aging and DNA damage-associated disease conditions in the future."

First draft submitted: 5 March 2019; Accepted for publication: 12 March 2019; Published online: 9 May 2019

Age is the main risk factor for many common noncommunicable diseases (NCDs), including diabetes mellitus, cancer, cardiovascular disease, osteoporosis and neurodegeneration [1]. Epigenomic modifications that are altered in the genomes of the elderly may initiate the cellular pathogenesis mechanism of NCDs [2]. Future medicine should explore whether reversal of these epigenomic changes will improve the health of those suffering from ageassociated NCDs. Reduction in intersperse repetitive sequence (IRS) methylation or global hypomethylation is commonly found in the elderly and NCDs, including diabetes mellitus, cancer, cardiovascular disease, osteoporosis and glaucoma [3-8]. Therefore, exploring whether and how global hypomethylation drives NCD pathogenesis and whether adding DNA methylation to IRSs improves NCD phenotypes would be interesting.

Two decades ago, global hypomethylation was proven to facilitate genomic instability processes or increase the rate of mutation [9]. However, how DNA methylation maintains genomic stability has not been elucidated. Previously, we found that Alu element methylation helps prevent DNA damage, an abnormal chemical structure in DNA [10]. Alu elements are a type of IRS with millions of copies dispersed throughout the human genome. We found an inverse association between the Alu element methylation level and DNA damage in white blood cells. When we increased Alu methylation levels in cultured cells by Alu siRNA, the transfected cells reduced endogenous DNA damage and improved resistance to DNA damaging agents [10]. Therefore, DNA methylation plays a role in maintaining genomic stability by preventing DNA damage.

Several lines of evidence suggest that spontaneous accumulation of DNA damage drives the aging process and NCD phenotypes [11]. For example, pathologic endogenous DNA double strand breaks (Path-EDSBs) are spontaneously produced and retained in chronological aging yeast [12]. Path-EDSBs were also detected indirectly as $\gamma \mathrm{H} 2 \mathrm{AX}$ foci in several organs of aging mammals, including humans [13-16]. Oxidative DNA damage refers to the oxidation of specific bases. 8-hydroxy-2'-deoxyguanosine (8-OHdG) is increased in cardiovascular patients and patients with diabetes mellitus, chronic lung disease, osteoporosis and neurological degeneration [11]. DNA damage may promote NCD phenotypes by promoting DNA damage repair (DDR) signal pathways. The role of DDR is to arrest cell cycle progression to allow for DNA repair and to prevent mutation due to DNA replication errors. However, too much DDR causes a number of pathogenic processes, including inflammation, metabolic rewiring, senescence, apoptosis and aging [17]. This may be the reason why the elderly have a delayed healing process. In addition to increasing Alu methylation and decreasing DNA damage, Alu siRNA-transfected cells increased cell proliferation [10]. Therefore, an increase in Alu methylation may reverse aging phenotypes and improve health conditions in NCD patients.

The molecular mechanism by which DNA methylation decreases DNA damage remains to be elucidated. Notably, the Alu siRNA experiment suggests that DNA methylation stabilizes the human genome in a long distance from the methylated sequence. While Alu siRNA increased Alu methylation levels by only $10 \%$, three-quarters of the endogenous 8-OHdG disappeared [10]. By rough estimation, DNA methylation of an Alu locus protected $30 \mathrm{~Kb}$ of

Future Medicine 
the human genome. Because this spreading effect does not harm cells, this modification should not directly interfere with gene expression. I propose a hypothesis that instead of spreading epigenomic modification or proteins and DNA interaction, DNA methylation relieves DNA tension and consequently stabilizes the genome in cis-acting up to several 10,000 base pairs of the genome [2].

Within the methylated genome, there is a type of lesser-known epigenetic marker that can relieve DNA tension [18]. These epigenetic markers are physiologic replication independent endogenous DNA double strand breaks (Phy-RIND-EDSBs) [18-20]. Because Phy-RIND-EDSBs are localized within the hypermethylated genome, global hypomethylated genomes possessed low levels of Phy-RIND-EDSBs [18]. Even though Phy-RIND-EDSBs are DSBs, Phy-RIND-EDSBs are epigenetic markers and not DNA damage lesions. Phy-RIND-EDSBs are found ubiquitously in all cells from yeast to humans $[18,19]$. Phy-RIND-EDSBs are reduced in cells lacking HMGB proteins and Sir2 [19]. This means eukaryotic cells have proteins that produce or maintain Phy-RIND-EDSBs. In yeast, cell viability correlated very strongly with Phy-RIND-EDSB levels $(r=0.94 ; p<0.0001)$ [20]. When Phy-RIND-EDSBs levels decreased, Path-EDSBs were augmented [20]. Therefore, Phy-RIND-EDSBs possess a physiologic function in maintaining genomic stability of the cells [20]. The role of Phy-RIND-EDSBs is similar to topoisomerase in that it relieves DNA torsion via physiologic EDSBs [19]. I hypothesized that Phy-RIND-EDSBs stabilize the genome by relieving DNA tension similar to tension reduction by gaps left in between rails of a railway track. Interestingly, Phy-RIND-EDSBs are reduced convincingly with age in chronological aging yeast [20]. Thus, Phy-RIND-EDSBs can be designated as youth-associated genomic-stabilizing DNA gaps [2].

In conclusion, the genome of the elderly is hypomethylated. Global hypomethylation increases genomic instability by increasing DNA damage. Too much DDR will halt the cell cycle and drive NCD pathogenesis. The increase in Alu methylation by Alu siRNA resulted in a reduction in DNA damage. Therefore, a technique adding IRS methylation, such as Alu siRNA transfection, may be useful for treating aging and DNA damage-associated disease conditions in the future.

\section{Author contributions}

A Mutirangura conceived of the presented idea, gathered information, developed the hypothesis and wrote the manuscript.

Financial \& competing interests disclosure

All studies in Thailand were supported by National Science and Technology Development Agency, The Thailand Research Fund and Chulalongkorn University, Thailand. The authors have no other relevant affiliations or financial involvement with any organization or entity with a financial interest in or financial conflict with the subject matter or materials discussed in the manuscript apart from those disclosed.

The article was language edited by American Journal Experts, no other writing assistance was utilized in the production of this manuscript.

\section{Open access}

This work is licensed under the Attribution-NonCommercial-NoDerivatives 4.0 Unported License. To view a copy of this license, visit http://creativecommons.org/licenses/by-nc-nd/4.0/

\section{References}

1. Licher S, Heshmatollah A, van der Willik KD et al. Lifetime risk and multimorbidity of non-communicable diseases and disease-free life expectancy in the general population: a population-based cohort study. PLoS Med. 16(2), doi: 10.1371/journal.pmed.1002741 (2019).

2. Mutirangura A. A hypothesis to explain how the DNA of elderly people is prone to damage: genome-wide hypomethylation drives genomic instability in the elderly by reducing youth-associated gnome-stabilizing DNA gaps. IntechOpen. (2018). www.intechopen.com /online-first/a-hypothesis-to-explain-how-the-dna-of-elderly-people-is-prone-to-damage-genome-wide-hypomethylation

3. Jintaridth P, Tungtrongchitr R, Preutthipan S, Mutirangura A. Hypomethylation of Alu elements in post-menopausal women with osteoporosis. PLoS ONE 8(8), doi: 10.1371/journal.pone.0070386 (2013).

4. Bollati V, Baccarelli A, Hou L et al. Changes in DNA methylation patterns in subjects exposed to low-dose benzene. Cancer Res. 67(3), 876-80 (2007).

5. Chalitchagorn K, Shuangshoti S, Hourpai N et al. Distinctive pattern of LINE-1 methylation level in normal tissues and the association with carcinogenesis. Oncogene 23(54), 8841-8846 (2004).

6. Wei L, Liu S, Su Z, Cheng R, Bai X, Li X. LINE-1 hypomethylation is associated with the risk of coronary heart disease in Chinese population. Arq. Bras. Cardiol. 102(5), 481-488 (2014). 
7. Jintaridth P, Mutirangura A. Distinctive patterns of age-dependent hypomethylation in interspersed repetitive sequences. Physiol. Genomics 41(2), 194-200 (2010).

8. Chansangpetch S, Prombhul S, Tantisevi V et al. DNA methylation status of the interspersed repetitive sequences for LINE-1, Alu, HERV-E, and HERV-K in trabeculectomy specimens from glaucoma eyes. J. Ophthalmol. 9171536, (2018).

9. Chen RZ, Pettersson U, Beard C, Jackson-Grusby L, Jaenisch R. DNA hypomethylation leads to elevated mutation rates. Nature 395(6697), 89-93 (1998).

10. Patchsung M, Settayanon S, Pongpanich M, Mutirangura D, Jintarith P, Mutirangura A. Alu siRNA to increase Alu element methylation and prevent DNA damage. Epigenomics 10(2), 175-185 (2018).

11. Milic M, Frustaci A, Del Bufalo A et al. DNA damage in non-communicable diseases: a clinical and epidemiological perspective. Mutat. Res. 776, 118-127 (2015).

12. Pongpanich M, Patchsung M, Mutirangura A. Pathologic replication-independent endogenous DNA double-strand breaks repair defect in chronological aging yeast. Front. Genet. 9, 501 (2018).

13. Siddiqui MS, Francois M, Fenech MF, Leifert WR. Persistent gammaH2AX: a promising molecular marker of DNA damage and aging. Mutat. Res. Rev. Mutat. Res. 766, 1-19 (2015).

14. Voss P, Siems W. Clinical oxidation parameters of aging. Free Radic. Res. 40(12), 1339-1349 (2006).

15. Poljsak B, Dahmane R. Free radicals and extrinsic skin aging. Dermatol. Res. Pract. doi: 10.1155/2012/135206 (2012).

16. Al-Mashhadi S, Simpson JE, Heath PR et al. Oxidative glial cell damage associated with white matter lesions in the aging human brain. Brain Pathol. 25(5), 565-574 (2015).

17. Ou HL, Schumacher B. DNA damage responses and p53 in the aging process. Blood 131(5), 488-495 (2018).

18. Pornthanakasem W, Kongruttanachok N, Phuangphairoj C et al. LINE-1 methylation status of endogenous DNA double-strand breaks. Nucleic Acids Res. 36(11), 3667-3675 (2008).

19. Thongsroy J, Matangkasombut O, Thongnak A, Rattanatanyong P, Jirawatnotai S, Mutirangura A. Replication-independent endogenous DNA double-strand breaks in Saccharomyces cerevisiae model. PLoS ONE 8(8), doi: 10.1371/journal.pone.0072706 (2013).

20. Thongsroy J, Patchsung M, Pongpanich M, Settayanon S, Mutirangura A. Reduction in replication-independent endogenous DNA double-strand breaks promotes genomic instability during chronological aging in yeast. FASEB J. doi: 10.1096/fj.201800218RR (2018). 
